\title{
Variability of sediment diatom assemblages of Lake Pont-de-Salars on the Viaur River (France)
}

\author{
S.O. Chung 1,2 \\ R. Le Cohu ${ }^{1}$
}

Keywords : diatoms, sediment, lake, relative abundance, biostratigraphy, Correspondence Analysis.

Limnological surveys from the pelagic zone of Lake Pont-de-Salars were undertaken during the 1993-1996 period, which included the emptying of the whole-lake in summer 1995 and the first post year refilling. This reservoir was warm and monomictic and was determined to vary between oligo- and meso-eutrophic status from year to year. Three sediment cores were collected from the deepest zone and frozen in situ during the short drying period, just after the emptying and before the refilling of the reservoir. Analysis of the relative abundance and vertical distribution of diatom taxa was undertaken. The diatom vertical distribution, evident by visual biostratigraphy, is confirmed by the correspondence analysis (CA) despite of absence of sediment dating.

Variabilité de l'assemblage de la flore diatomique des sédiments du Lac de Pont-de-Salars sur la rivière Viaur (France)

Mots-clés : diatomées, sédiments, lac, abondance relative, biostratigraphie, Analyse de Correspondance.

Quelques facteurs abiotiques et biotiques de la zone pélagique du lac de Pont-de-Salars ont été étudiés de 1993 à 1996, période incluant la vidange du réservoir (été 1995) et sa remise en eau. Ce réservoir est de type monomictique chaud et son statut trophique varie de oligotrophe à méso-eutrophe suivant l'année. Lors de la mise à sec, trois carottes de sédiment ont été effectuées dans une zone correspondant à la partie la plus profonde du lac et congelés «in situ». L'analyse de la répartition verticale des diatomées et de l'abondance relative des espèces a été effectuée. Les assemblages de diatomées mis en évidence par l'analyse biostratigraphique sont confirmés par une analyse de correspondance, malgré l'absence de datation du sédiment.

\section{Introduction}

Diatom assemblages preserved in lake sediments can directly reflect the diatom flora in the water column and can indirectly indicate lake water quality due to short generation times and sensitivity to their environment (Barttarbee 1986, Leavitt et al. 1994, Zeeb et al. 1994, Wessels et al. 1999). It is generally used in

1. Centre d'Ecologie des Systèmes Aquatiques Continentaux, UMR C5576, Université Paul-Sabatier, 118 route de Narbonne, 31062 Toulouse cedex, France.

2. Corresponding author : chung@cict.fr paleolimnology to reflect or reconstruct environmental changes despite the absence of past water chemistry data (Anderson 1989, Anderson 1990, Anderson et al. 1995, Smol 1990).

Initially the aim of this study was to observe the vertical variability of diatom assemblages in the recently accumulated sediments, which was supposed to reflect environmental conditions of the pelagic zone at that time (Feuillade et al. 1995). If there were some changes, it would be used to compare with diatoms in the pelagic zone before and after the emptying of the reservoir in order to observe the role of sediment diatoms in the recolonization of the water column (McQuoid \& Hobson 1995). 


\section{Study site}

Lake Pont-de-Salars is relatively large (200 ha) with a maximum depth of ca. $35 \mathrm{~m}$, a mean depth of ca. $11 \mathrm{~m}$, and a short water residence time of 66 days. It is located on the Viaur River and in the southwest of the Massif Central (Aveyron $44^{\circ} 16^{\prime} \mathrm{N}$ and $2^{\circ} 45^{\prime} \mathrm{E}$ ) in France at an altitude of $718 \mathrm{~m}$ (normal retention level). The lake's morphometric characteristics are presented in Table 1.

This non-hydropower reservoir built by Electricité de France (EDF) in the 1950's is upstream and is directly connected to the pumping station of Lake Bage downstream. The surface of Lake Pont-de-Salars is relatively well sheltered from wind because of the elevated (ca.720-804 m) and forested (mainly oak, pine and bushes) surrounding terrain.

The water body is long, narrow and slightly dendritic (Fig.1). The geological nature of the lake basin is mica schist and gneiss. Due to the geographical location, the climate has oceanic (spring and autumn), mediterranean (summer), and continental (winter) influences.

The major land use pattern of the sparsely populated (ca. 14 inhab. $/ \mathrm{km}^{2}$ ) surroundings ( $>70 \%$ ) is livestock breeding (bovine, ovine, porcine,...) and agriculture (cereals, feed grains and grasses). The reservoir is known for leisure activities (bathing, fishing, water sports and camping) in which untreated wastewater flows directly into the reservoir, in addition to the agricultural and dairy farm wastewaters and runoffs.

Since the construction of the reservoir in 1952, two whole-lake emptyings were performed : firstly in 1972 without monitoring and secondly during this study period of August 21-September 1, 1995.

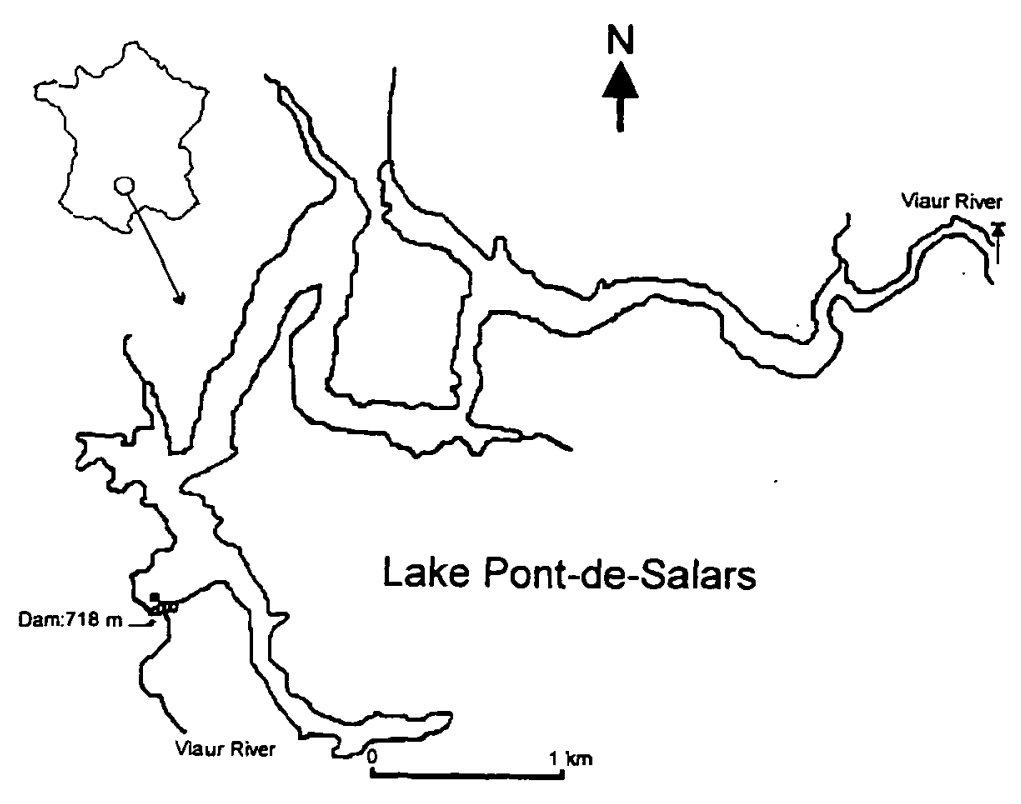

Fig. 1. Map of Lake Pont-de-Salars and the sediment sampling point.

Fig. 1. Situation géographique du Lac de Pont-de-Salars et localisation de l'échantillonnage des carottes de sédiment.

According to the sediment cores study of Lake Pareloup (Dagnac 1994), near to Lake Pont-de-Salars, yearly sediment accumulation thickness was estimated at $0.5 \mathrm{~cm}$ of dry deposits, and sediment laminae were well distinguished. However, once they were dried, sediment samples stayed intact, and could not be remoistened (Dagnac 1994). Moreover, according to the analyses of two sediment cores from the pelagic zone of Lake Pont-de-Salars (David 1998), the sediment was mainly composed of fine particles such as clay and silt (90\%) and contained about $81 \%$ of water, 12 to $17 \%$ of organic materials including $8 \%$ of organic carbon and $\mathrm{C}: \mathrm{N}$ ratio of 12 , which indicated the importance of resistant plants from the watershed and $1.7 \%$ of to-

Table 1. Morphometric characteristics of Lake Pont-de-Salars (Southwest, France).

Tableau 1. Caractéristiques morphométriques du Lac de Pont-de-Salars (Sud-Ouest, France).

\begin{tabular}{llll}
\hline Altitude & $718 \mathrm{~m}$ & Reservoir volume & $20.6 * 10^{6} \mathrm{~m}^{3}$ \\
Shore length & $50 \mathrm{~km}$ & Drainage area & $182 \mathrm{~km}^{2}$ \\
Surface area & $200 \mathrm{ha}$ & Maximum depth & $34.7 \mathrm{~m}$ \\
$\begin{array}{l}\text { Theoretical hydraulic } \\
\text { residence time }\end{array}$ & 65.5 days* & Mean depth & $11.3 \mathrm{~m}$ \\
\hline
\end{tabular}

* Period 1985-1994 
tal phosphorus (TP) which was higher than that of Lake Pareloup (David 1998). Suspended solids (SS) analyses at the time of emptying showed similar features, compared with those of the sediments, also a lot of accumulated organic materials of the sediments were supposed to be removed at this time as well (David 1998).

A summary of physical and chemical features of the pelagic zone in the whole water column before the emptying is presented in Table 2. The data characterize Lake Pont-de-Salars as unlimited (nutrients), mesotrophic (in particular, TP), modestly loaded ions (conductivity), crystalline (alkalinity), and usually neutral to slightly alkaline in the upper layer (down to metalimnion) in summer due to photosynthetic activities $(\mathrm{pH})$. Suspended solids concentrations varied widely from 0 to $37800 \mu \mathrm{g} \mathrm{l}^{-1}$. Secchi disc transparency was always above $3 \mathrm{~m}(<3 \mathrm{~m})$ which means that there was never an observed clear water phase before emptying. Chlorophyll $a$ concentrations reached a maximum in summer and varied widely (undetectable- 46 $\left.\mu \mathrm{g} \mathrm{l}^{-1}\right)$. The results seemed to reflect the influences of surrounding land use patterns and geological nature.
A trophic status of the reservoir was studied using the TP model (Vollenweider 1976), water residence time, chlorophyll $a$ and Secchi transparency (Fabre 1982) and some limnological studies (i.e., reservoir limnology, impact of inflow, biomanipulation, and phytoplankton dynamics...) were carried out during the 1993-1996 period (submitted \& in preparation).

\section{Material and methods}

To avoid the problems of representatives from using only one or two cores (Anderson 1989,1990), three short sediment cores $(16.5-18 \mathrm{~cm})$ from the pelagic zone were collected using hand handling PVC corers (inner diameter of $7 \mathrm{~cm}$ and $35 \mathrm{~cm}$ long) and were frozen in situ in September 1995. In the absence of undulations in laminae contrary to that of Lake Pareloup (Dagnac 1994), frozen sediments were continuously sectioned $0.5 \mathrm{~cm}$ in accordance with approximately supposed annual increments (Dagnac 1994) from 0 to 2.5 $\mathrm{cm}$ : A lamina at $10-10.5 \mathrm{~cm}$ was also sectioned, and each last lamina of $0.5 \mathrm{~cm}$ was sectioned from the end which varied from 16.5 to $18 \mathrm{~cm}$. The same depth laminae from each core were well mixed in order to

Table 2. Some physical and chemical characteristics of the water of Lake Pont-de-Salars before the emptying.

Tableau 2. Quelques paramètres physico-chimiques de l'eau du Lac de Pont-de-Salars avant le vidange.

Jul./Aug. 1993-Aug. 1995

Mean (min-max) $; \mu \mathrm{g}^{-1}$

$\mathrm{pH}$

Alkalinity $\left(\mathrm{CaCO}_{3} \mathrm{mg} \mathrm{1}^{-1}\right)$

Conductivity $\left(\mu \mathrm{S} \mathrm{cm}^{-1}\right)$

Suspended solids (SS)

Dissolved in organic nitrogen (DIN)

Total phosphorus (TP)

Silicate $(\mathrm{Si})$

Secchi disc transparency (SD : m)

Chlorophyll $a(\mathrm{Chl} a)$
$7.3(6.64-9.4)$

$24.6(11-80)$

91 (43-111)

$2368(0-37800)$

2024 (588-4175)

30 (5-808)

7869 (200-10950)

1.97 (1.3-2.85)

5.3 (undetectable-46) 
avoid heterogeneity (patchness) and were conserved in the cold room. Subsamples of $0.3 \mathrm{~g}$ per depth were prepared according to Barttarbee (1986). Cleaned and dried samples were embedded in Naphrax mounting medium. For each slide, at least 500 valves were counted using Nachet NS 400 light microscope (L.M. : oil immersion with 1200 times magnification). For further systematic identifications, the scanning electron microscopy (SEM) samples were prepared according to Le Cohu \& Coste (1995) and examined with a Cambridge Stereoscan $250 \mathrm{MK} 3$ the scanning electron microscope. The species or taxa were generally identified using Germain (1981), Krammer (1992), and Krammer \& Lange-Bertalot (1986, 1988, 1991a \&1991b). For taxonomic purposes and problems with small centrals such as Stephanodiscus, Cyclostephanos, and $C y$ clotella, we identified them according to published articles by Klee \& Schmidt (1987), Klee \& Houk (1996), Klee \& Casper (1997) and by personal communication with Klee in addition to precedent works of others (Chang 1991, Genkal \& Hakansson 1990, Hakansson \& Kling 1990).

For data analysis and presentation, we used percentage counting (relative abundance), biostratigraphy, and correspondence analysis which was used to summarize the variability of core biostratigraphy (Barttarbee 1986, Anderson 1989, 1990). Correspondence analysis was carried out using STATISTICA (StatSoft 2000).

\section{Results}

\subsection{Relative abundances and biostratigraphy}

All observed diatom taxa and relative abundance rating for the seven sediment laminae is presented in Table 3. 121 taxa were identified that belonged to 28 genera. 13 taxa were determined to genus only. 14 species have minimum relative abundances at more than $2 \%$ at least in one depth. $68-89 \%$ of the diatom assemblages belonged to the centrales (Cyclotella, $C y$ clostephanos, Stephanodiscus, Aulacoseira, Melosira) and Fragilariaceae (Asterionella, Diatoma, Fragilaria, Meridion, Tabellaria).

Fragilaria crotonensis was dominant ( $>20 \%$ of relative abundances), accompanied by either dominant or abundant ( $>5 \%$ of relative abundances) species such as Cyclotella radiosa, Stephanodiscus minutulus, and Asterionella formosa down to $2-2.5 \mathrm{~cm}$. For $C y$ clotella stelligeroides which was abundant in the top layer, it included blurred individuals which were very difficult to identify to species such as Cyclotella pseudostelligera, Cyclotella stelligera, and Cyclotella wol- tereckii. In the sediment samples of the $10-10.5 \mathrm{~cm}$, Asterionella formosa and Stephanodiscus minutulus were dominant accompanied by abundant Cyclotella stelligera and Aulacoseira subarctica which was also abundant at the $2-2.5 \mathrm{~cm}$ lamina. For the last layer $(16.5-18 \mathrm{~cm})$, Cyclotella stelligera was dominant, accompanied by abundant Tabellaria flocculosa, Achnanthes minutissima, and Fragilaria capucina which were never abundant above the laminae.

Vertical variability of mostly representative taxa was presented as a biostratigraphy in figure 2 which included the modestly recorded taxa occurring almost throughout the depths such as Gomphonema parvulum, Aulacoseira ambigua, Aulacoseira distans, and Navicula cryptocephala. The biostratigraphy summarized well the changes of the sediment depths and was in accordance with the rating of relative abundances (Table 3).

\subsection{Correspondence analysis}

For the CA ordination, 15 taxa were selected. 14 taxa were rated 'frequent' ( $>2 \%$ relative abundances at least in one layer) and Cyclotella pseudostelligera (relative abundance $>1.5 \%$ ) which could be confused with Cyclotella stelligeroides for the top layer (Fig. 3). Ordination of CA showed well distinguished vertical variabilities in four groups (about $89 \%$ of inertia mainly by X-axis of $72 \%$ ) to indicate the diatom assemblages in relation to sediment depths (L0-LL) as well as in accordance with relative abundances and biostratigraphy (Table 3 and Fig. 2). The top layer (L0-0.5 cm) was characterized by Stephanodiscus minutulus (SMi), Cyclotella pseudostelligera (CPs), Cyclotella stelligeroides (CSd), Asterionella formosa (AsF), and Aulacoseira subarctica (ASu). The following 4 layers (L0.5-1 cm, L1-1.5cm, $\mathrm{L} 1.5-2 \mathrm{~cm}$, and $\mathrm{L} 2-2.5 \mathrm{~cm}$ ) showed a very similar pattern associated with Cyclotella radiosa (CRa), Fragilaria crotonensis (FCr), and Cyclotella woltereckii (CWo) but they shared with the top layer Stephanodiscus minutulus (SMi), Aulacoseira subarctica (ASu), and Asterionella formosa (AsF). The 10-10.5 cm layer (L10) was well distinguished with respect to Aulacoseira ambigua (AAm), which had a high relative abundance value $(>2 \%)$ and thus was rated 'frequent' (Table 3) in this layer only, and shared with the top layer for Stephanodiscus minutulus (SMi), Cyclotella stelligera (CSt), Asterionella formosa (AsF), and Aulacoseira subarctica (ASu) and with the last layer, Achnanthes minutissima (AMi). The mixed last layer (LL) showed a sparsely dispersed pattern but was represented by Tabellaria flocculosa (TFl), Fragilaria 
Table 3. Diatom taxa list and relative abundance «rating» for the seven survey sediment layers : dominant $(\mathrm{D}>20 \%)$, abundant $(\mathrm{A}>$ $5 \%)$, frequent $(\mathrm{F}>2 \%)$, occasional $(\mathrm{O}>0,5 \%)$, present $(\mathrm{P})$, absent $(-)$.

Tableau 3. Liste des espèces de diatomées et les classes d'abondance relative pour les sept couches de sédiment étudiées : dominante $(\mathrm{D}>20 \%)$, abondante $(\mathrm{A}>5 \%)$, fréquente $(\mathrm{F}>2 \%)$, occasionnelle $(\mathrm{O}>5 \%)$, présente $(\mathrm{P})$, absente $(-)$

\begin{tabular}{|c|c|c|c|c|c|c|c|}
\hline Taxa name I sediment depth $(\mathrm{cm})$ & Lo-0. & $\mathbf{0 . 5}$ & $-1-1$ & L1.5-2 & $2-2.5$ & L10-10.5 & LLas \\
\hline Cyclotella glabriuscula (Grunow) Hakansson & - & - & - & - & $\vec{P}$ & - & - \\
\hline Cyclotella meneghiniana Kutzing & $\mathbf{P}$ & - & $\mathbf{P}$ & 0 & - & $\mathbf{P}$ & $\mathbf{P}$ \\
\hline Cyclotella pseudostelligera Hustedt & 0 & $\mathbf{P}$ & - & - & - & - & $p$ \\
\hline Cyclotella radiosa (Grunow) Lemmermann & A & A & $\mathbf{D}$ & A & A & $\mathbf{P}$ & $\mathbf{P}$ \\
\hline Cyclotella stelligera Cleve \& Grunow & 0 & $\mathrm{O}$ & 0 & 0 & $\mathrm{O}$ & A & $\mathrm{D}$ \\
\hline Cyclotella woltereckit Hustedt & F & A & $\mathbf{F}$ & $\mathbf{F}$ & $\mathbf{F}$ & 0 & $\mathbf{P}$ \\
\hline Cyclotella stelligeroides (Cyc.pseudostelligera, stelligera, and woltereckii) & A & - & $\mathbf{P}$ & - & - & $\mathbf{P}$ & $\mathbf{P}$ \\
\hline Cyclostephanos invisitatus (Hohn \& Hel.) Ther., Stoerm. \& Hak. & 0 & - & - & - & - & 0 & $\mathbf{P}$ \\
\hline Stephanodiscus hantzschii Grunow & 0 & 0 & 0 & 0 & 0 & 0 & - \\
\hline Stephanodiscus minutulus (Kützing) Cleve \& Möller & $\mathrm{D}$ & A & A & A & A & D & $\mathbf{P}$ \\
\hline Aulacoseira ambigua (Grunow) Simonsen & $\mathbf{P}$ & $\mathbf{P}$ & $\mathbf{P}$ & $\mathbf{P}$ & $P$ & $F$ & 0 \\
\hline Aulacoseira distans (Ehrenberg) Simonsen & $\mathbf{F}$ & $\mathbf{O}$ & - & $\mathbf{P}$ & $\mathbf{P}$ & - & $F$ \\
\hline Aulacoseira subarctica (O.Müller) Haworth & $\mathbf{F}$ & $\mathbf{F}$ & $\mathbf{F}$ & $\mathbf{F}$ & A & A & $\mathrm{O}$ \\
\hline Melosira varians Agardh & - & - & - & $\mathbf{P}$ & - & - & - \\
\hline Asterionella formosa Hassall & A & A & A & A & A & $\mathrm{D}$ & $\mathrm{F}$ \\
\hline Diatoma sp. & - & - & - & - & - & - & $\mathbf{P}$ \\
\hline Fragilaria arcus (Ehrenberg) Cleve & - & - & - & - & - & - & $\mathbf{O}$ \\
\hline Fragilaria capucina Desmazières & $P$ & $\mathbf{P}$ & $\mathbf{P}$ & $\cdot \mathbf{P}$ & $\mathbf{P}$ & $\mathrm{O}$ & A \\
\hline Fragilaria crotonensis Kitton & D & $\mathbf{D}$ & D & D & $\mathbf{D}$ & $F$ & $\mathbf{O}$ \\
\hline Fragilaria delicatissima (W.Smith) Lange-Bertalot & - & - & - & - & - & 0 & $\mathbf{P}$ \\
\hline Fragilaria parasitica(W.Smith) Grunow var subconstricta Grunow & - & - & - & - & $\mathbf{P}$ & - & - \\
\hline Fragilaria pinnata Ehrenberg & - & $\mathbf{P}$ & - & - & $\mathbf{P}$ & $\mathbf{P}$ & $P$ \\
\hline Fragilaria ulna (Nitzsch) Lange-Bertalot & $\mathbf{P}$ & $\mathbf{P}$ & $\mathbf{P}$ & $\mathbf{P}$ & $\mathbf{P}$ & $\mathbf{P}$ & $\mathbf{P}$ \\
\hline Fragilaria ulna var. acus (Kützing) Lange-Bertalot "Sippen" angustissima & - & $\mathbf{P}$ & $\mathbf{P}$ & $\mathbf{P}$ & $\mathbf{P}$ & $\mathbf{P}$ & $\mathbf{P}$ \\
\hline Meridion circulare (Greville) Agardh & - & $\mathbf{P}$. & - & - . & $\mathbf{P}$ & $\mathbf{P}$ & $\mathbf{P}$ \\
\hline Tabellaria flocculosa (Roth) Kützing & $\mathbf{P}$ & - & $\mathbf{P}$ & $\mathbf{P}$ & $P$ & 0 & A \\
\hline Achnanthes biasolettiana Grunow & - & $\mathbf{P}$ & - & - & - & - & - \\
\hline Achnanthes biasolettiana Grunow var.subatomus Lange-Bertalot & - & - & - & - & - & $P$ & - \\
\hline Achnanthes bioretii Germain & $\mathbf{P}$ & - & $\mathbf{P}$ & $\mathbf{P}$ & $\mathbf{P}$ & $\mathrm{P}$ & $\mathrm{O}$ \\
\hline Achnanthes clevei Grunow & - & $\mathbf{P}$ & $\mathbf{P}$ & - & - & - & - \\
\hline Achnanthes coarctata (Brébisson) Grunow & - & - & - & - & - & - & $\mathbf{P}$ \\
\hline Achnanthes daonensis Lange-Bertalot & - & $\mathbf{P}$ & - & - & - & - & - \\
\hline Achnanthes lanceolata (Brébisson) Grunow & $\mathbf{P}$ & $\mathbf{P}$ & $\mathbf{P}$ & $\mathbf{P}$ & 0 & 0 & $\mathrm{O}$ \\
\hline Achnanthes minutissima Kützing & $\mathbf{F}$ & $\mathbf{F}$ & $\mathbf{F}$ & $F$ & $F$ & $\mathrm{~F}$ & A \\
\hline Achnanthes oblongella Oestrup & - & - & - & - & - & $\mathrm{P}$ & - \\
\hline Achnanthes sp. & 0 & - & $\mathbf{P}$ & - & - & - & $\mathrm{O}$ \\
\hline Amphora pediculus Kützing & $\mathbf{P}$ & $\mathbf{P}$ & $\mathbf{P}$ & - & $\mathbf{P}$ & - & $\mathbf{P}$ \\
\hline Caloneis bacillum (Grunow) Cleve & - & - & - & - & - & - & $\mathbf{P}$ \\
\hline Caloneis silicula (Ehrenberg) Cleve & - & - & $\mathbf{P}$ & $\mathbf{P}$ & - & $P$ & - \\
\hline Cocconeis placentura Ehrenberg & - & - & - & - & - & $P$ & $\mathbf{P}$ \\
\hline Cocconeis placentura Ehrenberg var. euglypta (Ehrenberg) Grunow & - & $\mathbf{P}$ & - & $\mathbf{P}$ & - & - & - \\
\hline Cymbella affinis Kützing & - & $\mathbf{P}$ & - & $\mathbf{P}$ & $\mathrm{O}$ & $\mathbf{P}$ & $P$ \\
\hline Cymbella amphicephala Naegeli & - & - & - & - & - &. & $\mathbf{P}$ \\
\hline Cymbella cymbiformis Agardh & $\mathbf{P}$ & - & - & - & - & - & - \\
\hline Cymbella minuta Hilse & 0 & $\mathbf{P}$ & $\mathbf{P}$ & 0 & 0 & 0 & $\mathrm{O}$ \\
\hline Cymbella sinuata Gregory & $\mathbf{P}$ & $\mathrm{O}$ & 0 & $\mathbf{P}$ & $\mathbf{P}$ & $P$ & 0 \\
\hline Cymbella triangulum (Ehrenberg) Cleve & $\mathbf{P}$ & 0 & $\mathbf{P}$ & $\mathbf{P}$ & $\mathbf{P}$ & - & - \\
\hline Cymbella sp. & - & - & $\mathbf{P}$ & - & - & - & - \\
\hline Denticula elegans Kützing & - & $\mathbf{P}$ & $\mathbf{P}$ & 0 & $\mathrm{O}$ & - & - \\
\hline Denticula spp. & 0 & $\mathbf{P}$ & - & $\mathbf{P}$ & $\mathbf{P}$ & - & - \\
\hline Diploneis elliptica (Kützing) Cleve & - & $\mathbf{P}$ & - & $\mathbf{P}$ & $\mathrm{O}$ & - & - \\
\hline Diploneis parma Cleve & - & - & - & - & - & - & $P$ \\
\hline Diploneis petersenii Hustedt & - & - & - & - & -. & - & $\mathbf{P}$ \\
\hline Entomoneis ornata (Bailey) Reimer & $\mathbf{P}$ & - & - & - & $\mathbf{P}$ & $\mathbf{P}$ & - \\
\hline Eunotia bilunaris (Ehrenberg) Mills & - & - & - & $\mathbf{P}$ & - & - & 0 \\
\hline Eunotia implicata Nörpel et al. & - & - & - & $\mathbf{P}$ & - & - & - \\
\hline Eunotia paludosa Grunow & - & - & - & - & $\mathbf{P}$ & - & - \\
\hline Eunotia veneris (Kuitzing) De Toni & - & - & - & - & - & - & $\mathbf{P}$ \\
\hline
\end{tabular}


Table. 3. Continued.

Tableau. 3. Suite.

Eunotia spp.

Frustulia homboides (Ehrenberg) De Toni var. crassinervia (Brébisson) Ross

Gomphonema acuminatum Ehrenberg

Gomphonema angustum Agardh

Gomphonema constrictum Ehrenberg

Gomphonema olivaceum (Hornemann) Brébisson

Gomphonema parvulum Kutzing

Gyrosigma sp.

Navicula accomoda Hustedt

Navicula angustata W.Smith

Navicula atomus (Kützing) Grunow

Navicula bacillum Ehrenberg

Navicula capitata Ehrenberg

Navicula cocconeiformis Gregory

Navicula rryptocephala Kutzing

Navicula elginensis (Gregory) Ralfs

Navicula globulifera Hustedt

Navicula gregaria Donkin

Navicula krasskei Hustedt

Navicula lanceolata (Agardh) Ehrenberg

Navicula mutica Küzing

Navicula pupula Kutzing var. pupula

Navicula radiosa Kützing

Navicula mynchocephala Küzing

Navicula similis Krasseke

Navicula subrhynchocephala Hustedt

Navicula trivialis Lange-Bertalot

Navicula sp.

Neidium affine var. longiceps (Gregory) Cleve

Nitzschia acicularis (Kutzing) W.Smith

Nitzschia amphibia Grunow

Nitzschia bergii Cleve-Euler

Nitzschia capitellata Hustedt

Nitschia dissipata (Kutzing) Grunow

Nitzschia fonticola Grunow

Nitzschia fruticosa Hustedt

Nitzschia hantzschiana Rabenhorst

Nitzschia heufleuriana Grunow

Nitzschia holsatica Hustedt

Nitzschia palea (Kutzing) W.Smith

Nitschia aff.paleacea Grunow

Nitzschia pellucida Grunow

Nitzschia aff.pseudofonticola Hustedt

Nitzschia aff.sublinearis Hustedt

Nitzschia tubicola Lange-Bertalot

Nitzschia sp. 1

Nitzschia sp. 2

Nitzschia sp.3

Pinnularia accuminata W.Smith

Pinnularia borealis Ehrenberg

Pinnularia interrupta W.Smith

Pinnularia lundii Hustedt

Pinnularia microstauron (Ehrenberg) Cleve

Pinnularia obscura Krasske

Pinnularia viridis (Nitzsch) Ehrenberg

Pinnularia spp.

Stauroneis phoenicenteron (Nitzsch) Ehrenberg

Surirella angusta Kützing

Surirella ovalis Brébisson

Suriralla roba Leclercq

Surirella splendida (Ehrenberg) Kützing

Surirella spp. 
Asterionella formosa

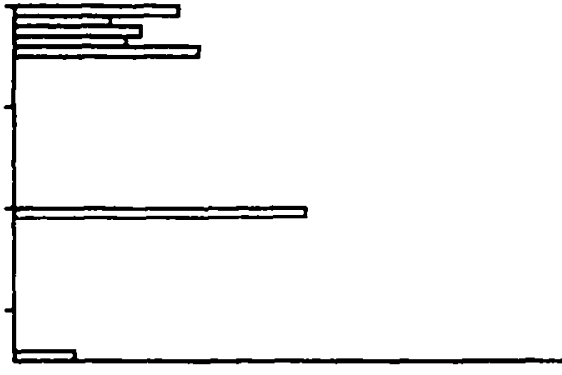

Stephanodiscus minutulus

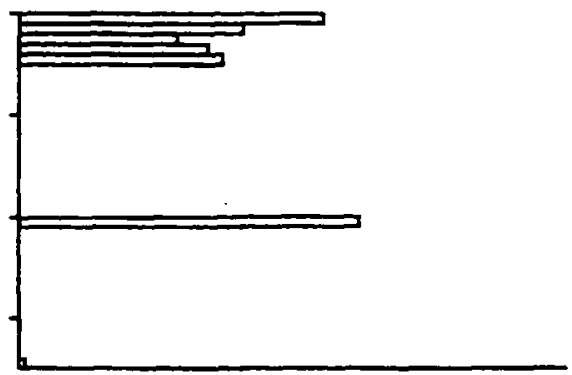

Cyclotella stelligera

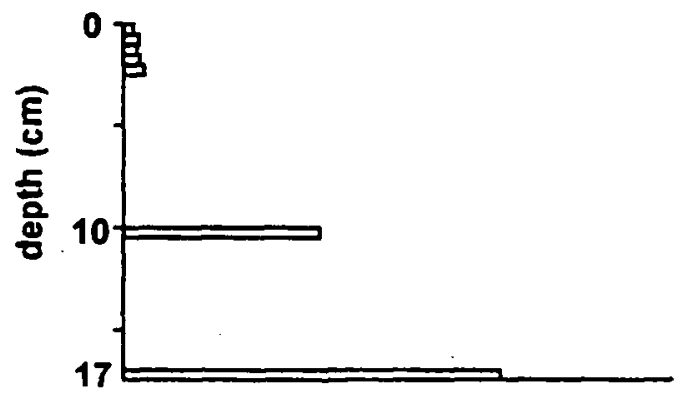

Cyclotella radiosa

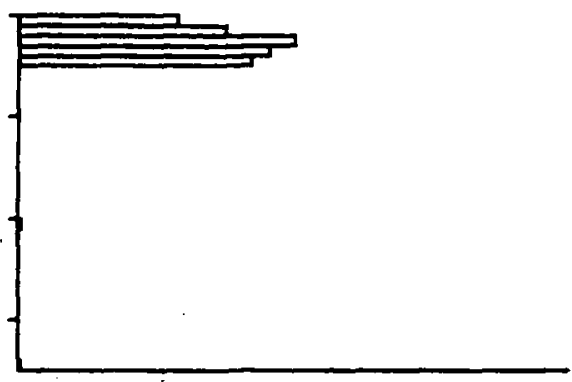

Fragilaria crotonensis

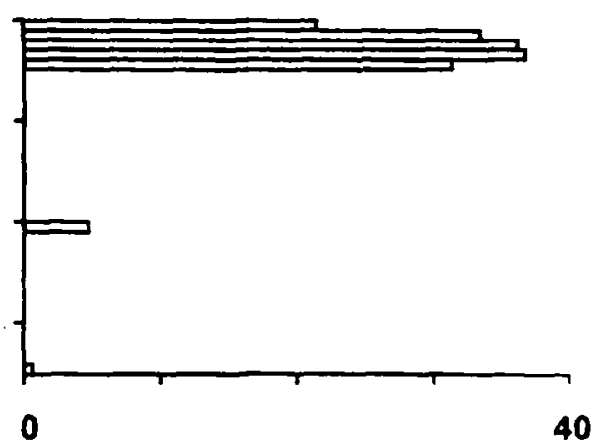

Cyclotella woltereckii

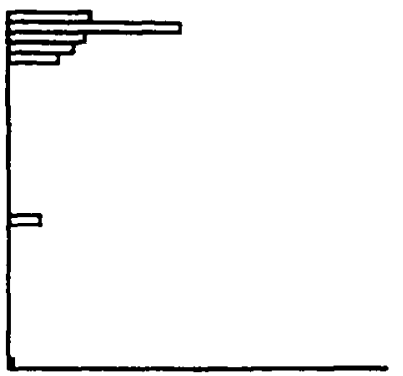

Fragilaria capucina

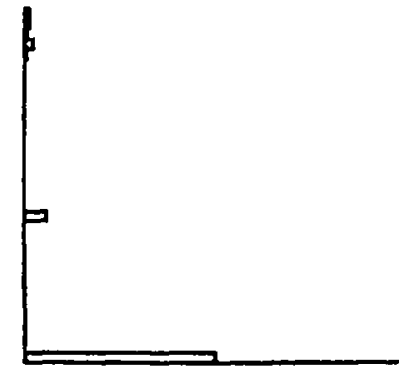

Aul. subarctica

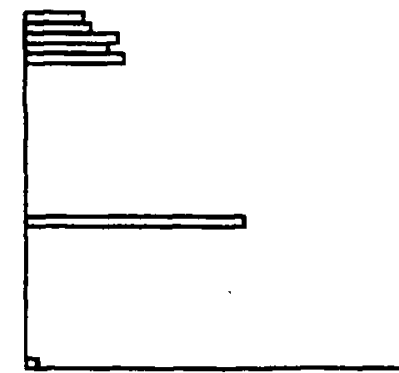

Ach. minutissima

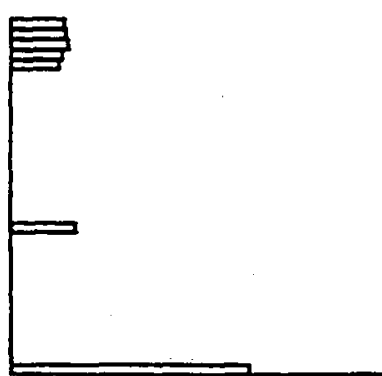

Tabellaria flocculosa

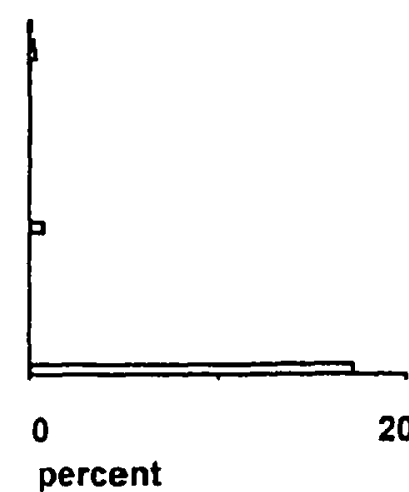

Nav.cryptocephala

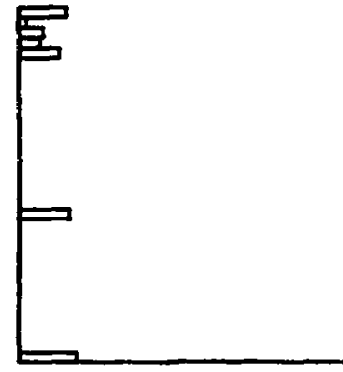

Aul.ambigua

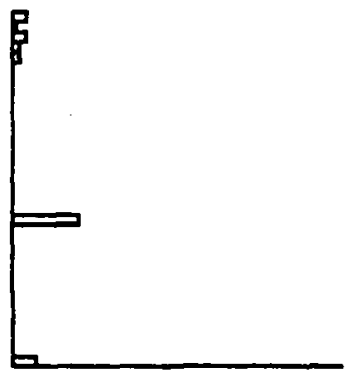

Gom.parvulum

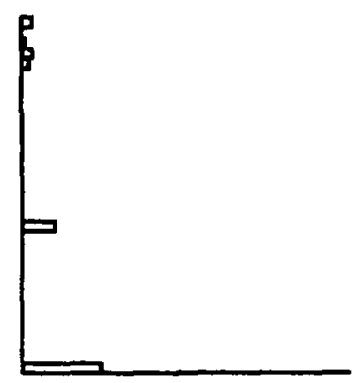

Aul.distans

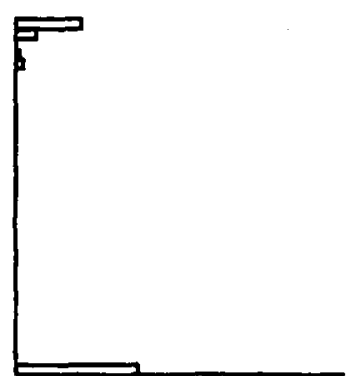

Cyc.stelligeroides

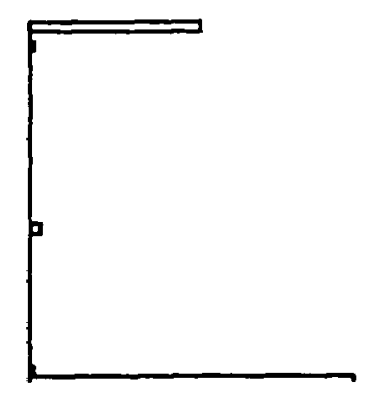

Fig. 2. Summary of diatom biostratigraphy for 15 representative taxa.

Fig. 2. Répartition verticale de 15 espèces représentatives. 


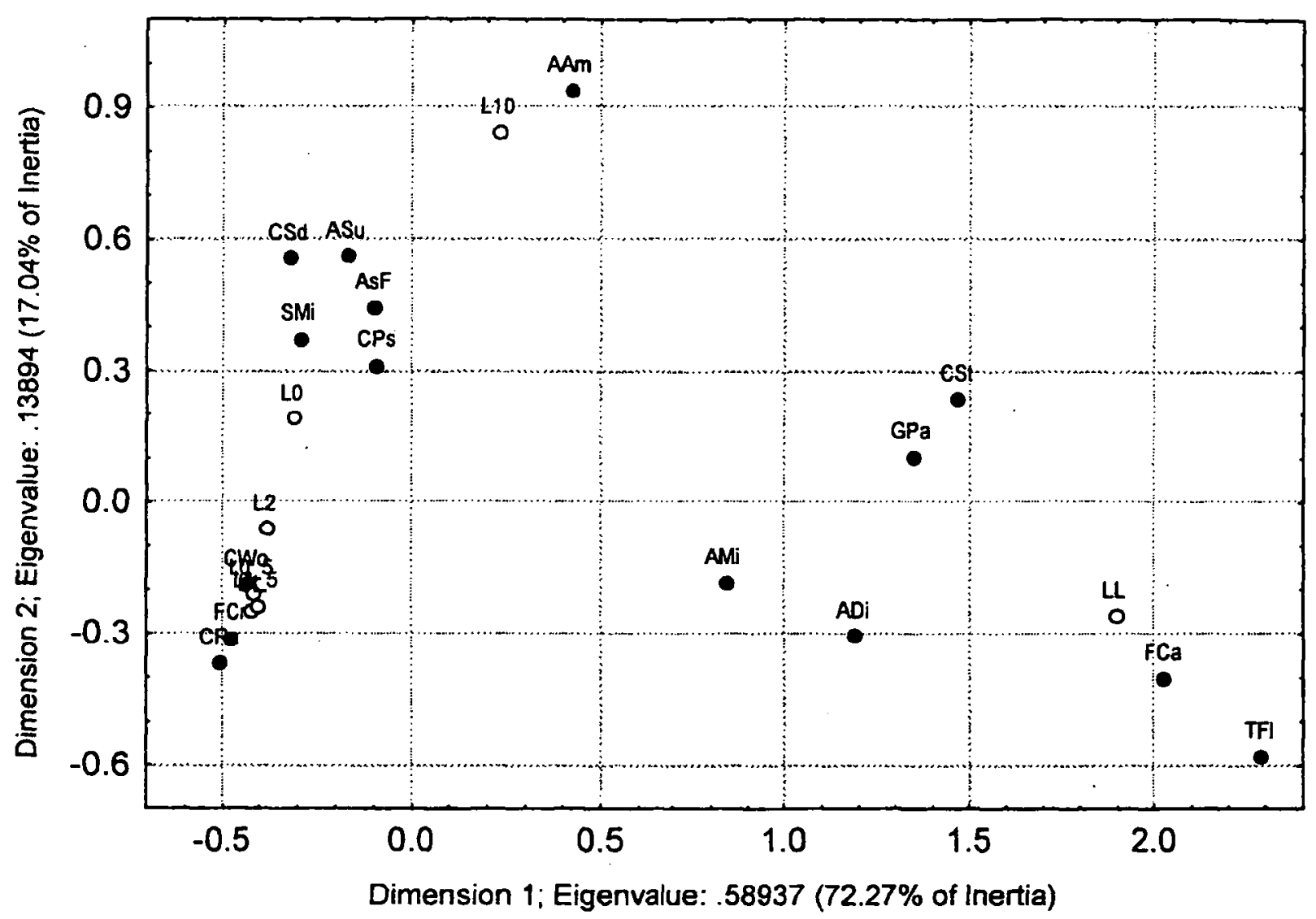

Fig. 3. CA ordination for 15 representative taxa in relation to the layer : $L 0-0.5 \mathrm{~cm}, L 0.5-1 \mathrm{~cm}, \mathrm{~L} 1-1.5 \mathrm{~cm}$, $\mathrm{L} 1.5-2 \mathrm{~cm}, \mathrm{~L} 2-2.5 \mathrm{~cm}, \mathrm{~L} 10-10.5 \mathrm{~cm}$, and LL ; the three mixed last laminae of $16.5-18 \mathrm{~cm}$. Stephanodiscus minutulus (SMi), Cyclotella pseudostelligera (CPs), Cyclotella stelligeroides (CSd), Asterionella formosa (AsF), Aulacoseira subarctica (ASu), Cyclotella radiosa (CRa), Fragilaria crotonensis (FCr), Cyclotella woltereckii (CWo), Aulacoseira ambigua (AAm), Cyclotella stelligera (CSt), Achnanthes minutissima (AMi), Tabellaria flocculosa (TFl), Fragilaria capucina (FCa), Aulacoseira distans (ADi), and Gomphonema parvulum (GPa).

Fig. 3. Ordination de l'analyse de correspondance pour 15 taxons représentatifs en fonction des couches : L0-0.5 cm, L0.5-1 cm, L1-1.5 cm, L1.5-2 cm, L2-2.5 cm, L10-10.5 cm, and LL ; les trois dernières laminae mélangées de $16.5-18 \mathrm{~cm}$.

capucina (FCa), Achnanthes minutissima (AMi), Aulacoseira distans (ADi), Gomphonema parvulum (GPa), and Cyclotella stelligera (CSt) which were distinguished by highest relative abundances in this layer only.

\section{Discussion and conclusion}

\subsection{Diatom assemblage changes}

Vertical variability of diatom assemblages indicated little change between the top layer (LO) and the (supposed) recently accumulated sediments (L0.5-L2) which showed the same type of diatom assemblages using relative abundances, thereafter illustrated by the biostratigraphy, and finally by the correspondence analysis. However, the discontinuities or changes of dia- tom assemblages with sediment depths were clearly observed in the L10 and LL layers which were discontinuously sliced from the top layers. The sparsely dispersed pattern of the last layer may reflect the heterogeneity of the sediment preparation due to different depths among the three cores $(16.5-18 \mathrm{~cm})$.

The taxa rating such as dominant, abundant, and frequent (Table 3 ) in the supposed recently accumulated sediments $(0-2.5 \mathrm{~cm})$ were generally observed with relatively important proportions in the pelagic zone before the emptying (in preparation). The importance of centrales (Cyclotella, Cyclostephanos, Stephanodiscus, Aulacoseira, and Melosira) and Fragilariaceae (Asterionella, Diatoma, Fragilaria, Meridion, and Tabellaria) was in accordance with Wessels et al. (1999) in Lake Constance, in particular. 


\subsection{Trophic status changes in relation to diatom as- semblages}

We would try to speculate or reconstruct the nutrient levels in the pelagic zone for the diatom assemblages from the sediment depths (i.e., Bennion et al. 2000, Lange-Bertalot \& Steindorf 1996, Feuillade et al. 1995), despite the absence of both sediment dating, which is used to determine the time of sediment accumulations, and sediment analyses (Anderson 1989, Anderson 1990, Barttarbee 1986, Dixit \& Evans 1986, Feuillade et al. 1995, Wessels et al. 1999).

In general, dominant, abundant, and frequent taxa of all the observed layers (Table 3 ) were generally characterized in the category of 'eutrophic' and/or 'tolerant' which means they widely occured from oligo- to eutrophic status such as well known Asterionella formosa and Fragilaria crotonensis (Lange-Bertalot \& Steindorf 1996, Feuillade et al. 1995).

The replacement of relatively important taxa from the last layer (Cyclotella stelligera, Tabellaria flocculosa, Fragilaria capucina, and Achnanthes minutissi$\mathrm{ma}$ ) to $10-10.5 \mathrm{~cm}$ layer by Stephanodiscus minutulus, Asterionella formosa, Aulacoseira subarctica and the diminution of importance of Cyclotella stelligera (from 28 to $14 \%$ ) indicated a nutrient level shift from relatively low to moderate from the time of the last layer to the moment of $10-10.5 \mathrm{~cm}$ layer (Bennion et al. 2000). The relative abundance of Stephanodiscus minutulus, Asterionella formosa, and Aulacoseira subarctica were highest at the layer of $10-10.5 \mathrm{~cm}$ among the layers. For Cyclotella radiosa and Fragilaria crotonensis which occurred firstly, there are remarkable amounts and then persistent abundances or dominances (Table 3) up to the top layer from the $2-2.5 \mathrm{~cm}$ layer. Otherwise, it means pronounced species shifts from the $10-10.5 \mathrm{~cm}$ layer to this layer but their trophic status varied widely from oligo- to eutrophic by these assemblages (Bennion et al. 2000, Lange-Bertalot \& Steindorf 1996, Feuillade et al. 1995). We already have discussed the similarity of species assemblages in the four adjacent layers from $2.5 \mathrm{~cm}$ to $0.5 \mathrm{~cm}$ above. Little change of species assemblages were noted due to a modest increase of Stephanodiscus minutulus from these layers to the top layer of $0-0.5 \mathrm{~cm}$.

\subsection{Case of Cymbella triangulum (Ehrenberg) Cleve}

Recently noted as an invading and expanding species in France and Europe (Bertrand \& Coste 1994), Cymbella triangulum only occurred modestly (Table 3) down to $2.5 \mathrm{~cm}$ in the sediment cores which seemed in accordance with this hypothesis.
In summary, despite the absence of sediment dating and sediment analyses, the diatom assemblages of the sediment cores showed vertical variability with the depths using relative abundances, biostratigraphy, and correspondence analysis from the pelagic zone of Lake Pont-de-Salars.

First, each layer was represented by four or five dominant or abundant taxa composed mainly by centrales (Cyclotella, Cyclostephanos, Stephanodiscus, Aulacoseira, Melosira) and Fragilariaceae (Asterionella, Diatoma, Fragilaria, Meridion, Tabellaria).

Secondly, four distinguished diatom groups with sediment depths were observed by the $\mathrm{CA}$ in accordance with relative abundances; the top layer ( $\mathrm{LO})$, the next following four layers (L0.5-L2), the $10-10.5 \mathrm{~cm}$ layer (L10), and the last layer (LL). From the last layer to the top layer, diatom assemblages reflected moderately and continuously nutrient enriched conditions. However, it showed little environmental change between the top layer and the four following layers. The occurrence of Cymbella triangulum down to $2.5 \mathrm{~cm}$ only seemed to indicate that the first layers (L0-L2.5) were recently accumulated. Diatom assemblages of the $10-10.5 \mathrm{~cm}$ and the last layer showed differences compared with the top layers. The last layer showed a very distinctive and dispersed pattern, with characteristic diatom taxa (Cyclotella stelligera, Tabellaria flocculosa, Fragilaria capucina, Achnanthes minutissima) which indicated indirectly both a shift of environmental conditions and the heterogeneity of sample preparations.

\section{Acknowlegements}

We thank the Drs Joseph Dagnac ${ }^{\dagger}$ and Cécile David for the sediment samplings and also Y.S. Park for statistical help and useful discussions.

\section{References}

Anderson N.J. 1989. - A whole-basin diatom accumulation rate for a small eutrophic lake in Northern Ireland and its palaeological implications. J. Ecology, 77 : 926-946.

Anderson N.J. 1990. - Variability of sediment diatom assemblages in an upland, wind-stressed lake (Loch Fleet, Galloway, S.W. Scotland). J. Paleolimnology, $4: 43-59$.

Anderson N.J., Renberg I. \& Segerström U. 1995. - Diatom production responses to the development of early agriculture in a boreal forest lake-catchment (Kassjön, northern Sweden). J. Ecology., $83: 809-822$.

Battarbee R.W. 1986. - Diatom analysis. In Berglund B.E. (ed.) Handbook of Holocene palaeoecology and palaeohydrology, J. Wiley \& Sons. Chichester : 527-568.

Bennion H., Monteith D. \& Appleby P. 2000. - Temporal and geographical variation in lake trophic status in the English Lake District : evidence from (sub)fossil diatoms and aquatic macrophytes. Freshwat. Biol., 45 : 394-412. 
Bertrand J. \& Coste M. 1994. - Cymbella triangulum (Ehrenb.) Cleve (Bacillariophyceae), un taxon nouveau pour la flore européenne ; caractéristiques morphologiques et écologiques. Cryptogamie, Algol., 15 : 147-158.

Chang T.P. 1991. - Elecktronenmikroskopische Untersuchungen an dem Typusmaterial der zentrischen Kieselalge Cyclotella stelligera Cleve et Grunow. Diatom Res., 6 : 1-14.

Dagnac J. 1994. - Bilan des dépôts sédimentaires dans le réservoir de Pareloup (Aveyron, France). Hydroécol. Appl., 6 : 59-85.

David C. 1998. - Caractérisation des matières en suspension lors d'événements hydrologiques particuliers. Thèse, Univ. Paul Sabatier Toulouse : 249 p.

Dixit S.D. \& Evans R.D. 1986. - Spatial variability in sedimentary algal microfossils and its bearing on diatom-inferred $\mathrm{pH}$ reconstruction. Can. J. Fish. Aquat. Sci., 43 : 1836-1845.

Fabre A. 1982. - Application d'un modèle de risque d'eutrophisation à un échantillon de réservoirs. CNRS.CERR.Toulouse : $32 \mathrm{p}$.

Feuillade M., Dominik J., Druart J.-C. \& Loizeau J.-L. 1995. - Trophic status evolution of Lake Nantua as revealed by biological records in sediment. Arch. Hydrobiol., 132 : 337-362.

Genkal S.I.\& Hakansson H.1990. - The problem of distinguishing the newly described diatom genus Pseudostephanodiscus SIEMICSKA. Diatom Res., 5 : 15-23.

Germain H. 1981. - Flore des Diatomées. N. Boubée, Paris : $444 \mathrm{p}$.

Hakansson H. \& Kling H. 1990. - The current status of some very small freshwater diatoms of the genera Stephanodiscus and $C y$ clostephanos. Diatom Res., 5 : 273-287.

Klee R. \& Schmidt R. 1987. - Eutrophication of Mondsee (upper Austria) as indicated by the diatom stratigraphy of a sediment core. Diatom Res., 2 : 55-76.

Klee R. \& Houk V. 1996. - Morphology and ultrastructure of $C y$ clotella woltereckii Hustedt (Bacillariariophyceae). Arch. Protistenkd, 147 : 19-27.

Klee R. \& Casper J. 1997. — Once more : Kützing's Type material of Stephanodiscus minutulus (Kütz.) Grunow (Bacillariophyceae) from «Liuneburg»-a Reinvestigation. Arch. Protistenkd, 148 : 53-63.

Krammer K. \& Lange-Bertalot H. 1986. - Bacillariophyceae Teil1 : Naviculaceae. Die Süßwasserflora von Mitteleuropa, Ettl H., Gerloff J., Heynig H. \& Mollenhauer D. (eds.) ; 2/1 : G. Fisher Verlag., Stuttgart : 876 p.
Krammer K. \& Lange-Bertalot H. 1988. - Bacillariophyceae Teil 2 : Bacillariaceae, Epithemiaceae, Surirellaceae. Die Süßwasserflora von Mitteleuropa 2/2, Ettl H., Gerloff J., Heynig H. \& Mollenhauer D. (eds.) : G. Fisher Verlag., Stuttgart : $596 \mathrm{p}$.

Krammer K. \& Lange-Bertalot H. 1991a. - Bacillariophyceae Teil 3 : Centrales, Fragilariaceae, Eunotiaceae. Die Süßwasserflora von Mitteleuropa 2/3, Ettl H., Gerloff J., Heynig H. \& Mollenhauer D. (eds.) ; $2 / 3$ : G. Fisher Verlag., Stuttgart : $576 \mathrm{p}$.

Krammer K. \& Lange-Bertalot H. 1991b. - Bacillariophyceae Teil 4 : Achnantheceae, Kritische Ergänzungen zu Navicula (Lineolatae) und Gomphonema. Die Süßwasserflora von Mitteleuropa 2/2, Ettl H., Gerloff J., Heynig H. \& Mollenhauer D. (eds.) : G. Fisher Verlag., Stuttgart : $596 \mathrm{p}$.

Krammer K. 1992. - Pinnularia, eine Monographie der europäischen Taxa. Bibl. Diatomol. $26: 353$ p.

Lange-Bertalot H. \& Steindorf A. 1996. - Rote list der limnischen Kieselalgen Bacillariophyceae. Deutschlands. Schr.-R.f. Vegetationskde, $28: 633-677$.

Leavitt P.R., Hann B.J., Smol J.P., Zeeb B.A., Christie C.E., Wolfe B. \& Kling H.J. 1994. - Paleolimnological analysis of whole-lake experiments : an overview of results from experimental lakes area lake 227. Can. J. Fish. Aquat. Sci., 51 : 2322-2332.

Le Cohu R. \& Coste M. 1995. — Le genre Gomphoneis (Bacillariophyta) : un nouveau modèle d'organisation du cingulum. Can. J. Bot., $73: 112-120$.

McQuoid M.R. \& Hobson L.A. 1995. - Importance of resting stages in diatom seasonal succession. J. Phycol., $31: 44-50$.

Smol J.P. 1990. - Are we building enough bridges between paleolimnology and aquatic ecology ? Hydrobiologia, 214 : 201-206.

StatSoft Inc. 2000. - STATISTICA for Windows [Computer program manual]. Tulsa, OK. U.S.A.

Vollenweider R.A. 1976. - Advances in defining critical loading levels for phosphorus in lake eutrophication. Mem. Ist. Ital. Idrobiol., $33:$ 53-83.

Wessels M., Mohaupt K, .Kümmerlin R. \& Lenhard A. 1999. - Reconstructing past eutrophication trends from diatoms and biogenic silica in the sediment and the pelagic zone of Lake Constance, Germany. J. Paleolimnology, $21: 171-192$.

Zeeb B.A., Christie C.E., Smol J.P., Findlay D.L., Kling H.J. \& Birks H.J.B. 1994. - Responses of diatom and chrysophyte assemblages in Lake 227 sediments to experimental eutrophication. Can. J. Fish. Aquat. Sci., 51 : 2300-2311. 